\title{
No App is an Island: Collective Action and Sustainable Development Goal-Sensitive Design
}

\author{
Steph Pitt, Marlína van Meelis Lacey, Ed Scaife, Jeremy Pitt* \\ Imperial College London, London (UK) \\ Received 30 November 2020 | Accepted 20 December 2020 | Published 15 February 2021
}

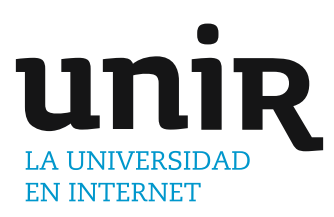

KEYWORDS

The transformation to the Digital Society presents a challenge to engineer ever more complex socio-technical systems in order to address wicked societal problems. Therefore, it is essential that these systems should be engineered with respect not just to conventional functional and non-functional requirements, but also with respect to satisfying qualitative human values, and assessing their impact on global challenges, such as those expressed by the UN sustainable development goals (SDGs). In this paper, we present a set of sets of design principles and an associated meta-platform, which focus design of socio-technical systems on the potential interaction of human and artificial intelligence with respect to three aspects: firstly, decision-support with respect to the codification of deep social knowledge; secondly, visualisation of community contribution to successful collective action; and thirdly, systemic improvement with respect to the SDGs through impact assessment and measurement. This methodology, of SDG-Sensitive Design, is illustrated through the design of two collective action apps, one for encouraging plastic re-use and reducing plastic waste, and the other for addressing redistribution of surplus food. However, as with the inter-connectedness of the SDGs, we conclude by arguing that the inter-connectedness of the Digital Society implies that system development cannot be undertaken in isolation from other systems.

\author{
Socio-Technical \\ Systems, Value-Sensitive \\ Design, Collective \\ Action, Plug-in \\ Architecture, Sustainable \\ Development Goals.
}

\section{INTRODUCTION}

$\mathrm{T}$ RANSITIONING to the Digital Society, as envisaged through the Digital Transformation, involves the increasing use of digi tal tools and technologies in the reconfiguration of business processes, organisational structures, and commercial transactions, and the recasting of social processes, physical infrastructure and relational interactions. This is having a profound impact on the nature of ownership (e.g. possession of goods being replaced by subscription to services), the sense of belonging to communities, and citizen's access to infrastructure for education, water, energy, medical treatment, and transportation; systems of manufacture, delivery and disposal; systems of justice, governance and political engagement; and monitoring and control over communal resources and the local environment.

In this context, there is a challenge to engineer ever more complex socio-technical and cyber-physical systems to support and enhance this full spectrum of human activities, to address wicked societal problems. Therefore, as outlined in Section II, it is essential that these systems should be engineered with respect not just to conventional functional and non-functional requirements, but also with respect to satisfying qualitative human values, and assessing their impact on global challenges, such as those expressed by the UN sustainable development goals (SDGs), a set of inter-connected goals intended to achieve a fairer and more inclusive future world.

${ }^{*}$ Corresponding author.

E-mail address: j.pitt@imperial.ac.uk
To meet this challenge, this paper builds on the methodologies of Value-Sensitive Design [1] and Socially-Sensitive Design [2], and proposes a methodology of SDG-Sensitive Design. To begin with, Section III considers a range of different value-sensitive design perspectives on engineering a socio-technical system. Here, we consider a socio-technical system to be one which recognises the importance of interaction between people and technology in system design: crucially, in the Digital Society that technology includes components with Artificial Intelligence, as manifested by a software agent, 'smart' device, robot, and so on.

From each perspective, Section III.C derives a set of sets of design principles, which focus design of socio-technical systems on the potential interaction of human and artificial intelligence with respect to three aspects: firstly, decision-support with respect to the codification of deep social knowledge; secondly, visualisation of community contribution to successful collective action; and thirdly, systemic improvement with respect to the SDGs through impact assessment and measurement. In Section IV, an associated meta-platform is described, whose key features include transparency, generativity and reconfiguration through plugins, which can be used to encode the deep social knowledge encapsulated in the design principles, and to evaluate, measure and visualise the contribution to achieving one or more of the SDGs.

This methodology is illustrated through the design of two collective action apps for sustainability, each targeted at a specific societal problem; one (described in Section V) for encouraging plastic reuse and reducing plastic waste, and the other (presented in Section VI) for addressing redistribution of surplus food. However, as with the inter- 
connectedness of the SDGs, we conclude by arguing that the interconnectedness of the Digital Society implies that no app is an island: system development cannot be undertaken in isolation from other systems, without concern for qualitative human values, or without considering its impact on achieving the SDGs.

\section{Problems, GoAls AND Impacts}

\section{A. Wicked Problems}

There are a host of societal problems that need to be addressed as part of the Digital Transformation to the Digital Society. This includes energy poverty, food insecurity, air quality, social justice and plastic reduction. However, all of these problems can be classified as wicked problems.

A wicked problem can be identified as a societal problem whose complexity and continually changing requirements is such that there is not necessarily a stopping rule or terminating condition, nor may there be a consistent set of criteria by which to evaluate immediately or ultimately such a condition. Additionally it may exhibit some or all of the other characteristics identified in [3].

The difficulty of finding digital solutions to wicked problems, like those listed above, is further exacerbated by (at least) five issues. Firstly, evaluation: for example, in the case of sustainability, there is no end state, by definition; so it can be asserted "has been sustained", and it can be claimed "is sustainable", but neither of these imply "will be sustained (indefinitely)"). Secondly, dealing with unexpected, emergency and potentially catastrophic situations, for which the system may not even have been designed. Thirdly, polycentricity: there being multiple stakeholders with different and possibly conflicting objectives. Fourthly, the potential of technologies like Artificial Intelligence (AI) to be mis-used, for example by creating intrusive monitoring frameworks (e.g. surveillance capitalism [4]). And finally, but perhaps above all, satisficing (rather than satisfying) qualitative human values - i.e. we are dealing with a multi-criteria sub-optimisation problem with subjective, non-numeric data points (cf. [5]).

This implies that system design is not restricted to functionality: it also has to take into account values, and other systems affecting the same values. Our approach consists of applying a set of design principles to develop systems for a new platform for social coordination - and then try to identify which of the SDGs it might impact, and consider how to measure that impact.

\section{B. Sustainable Development Goals (SDGs)}

Adopted by all United Nations (UN) Member States in 2015, the UN SDGs are a set of 17 interconnected goals to address fundamental global challenges by 2030, and have therefore been described as "the blueprint to achieve a better and more sustainable future." Designed to be inclusive of all, these goals are a call for action by all countries to address challenges related to poverty, inequality, the climate crisis, peace and justice. Importantly, SDG 17 calls for "Partnership for the Goals" which specifically highlights the importance of achieving each goal in concert with the others. These integrated and universally accepted goals underpin a shared agenda, therefore enabling a collective action response that encourages innovation, in which the transition to the Digital Society will play a profound role. More often, global societal challenges are being addressed through technological innovation, thus are likely to be making an impact on achieving one or more of the SDGs. Therefore, when considering how digital innovation can aid in achieving one, or indeed any, of the SDGs, identifying and measuring the impact made is crucial. Commonly referred to as impact measurement, having a quantifiable understanding of exactly how anything - in this case digital innovation - is shaping our progression towards achieving the SDGs is fundamental to appreciating where we currently stand, and what more needs to be done.

\section{Impact Measurement}

As mentioned above, the SDGs are designed to be achieved in concert, so whilst it is crucial to measure the positive impacts made to achieving some of the SDGs, it is equally important to measure unintended (and potentially negative) impacts against others, thereby enabling a holistic and fully comprehensive overview of projects, policies, and plans, particularly concerning digital innovation. However, it is equally important to operationalise targets and take into account the significance and relevance of specific metrics [6] not forgetting Goodhart's Law, that if any indicator or metric of some property of system is used instead as a target, then it ceases to function as a meaningful indicator of that property.

Beyond knowing how close (or not) a policy is to achieving its goals, impact measurement is both beneficial and important in many other respects. Firstly, it can aid with monitoring risks, as a desirable output does not necessarily lead to a desirable outcome. Secondly, impact measurement is crucial for business investment. According to [7], investors desire more detailed social and environmental performance data as this provides an improved understanding of non-financial returns, thus providing the opportunity for investment capital to be re-allocated accordingly. Thirdly, measuring impact enables accountability and transparency as it can be used as a metric to keep track of performance. Fourthly, impact measurement aids in better understanding social innovation. If social innovation is considered to be essentially experimental, we require impact measurement to inform the extent of success of such experiment. Finally, impact measurement offers a way to better communicate ideas, share views and add valuable contributions in a universally recognisable language, as the SDGs are stable and well-established.

However, there are a number of challenges to successful impact measurement, and despite being a noble expression of intention and ambition, ambiguity of interpretation generates a high level of complexity when attempting to measure impact. Primarily, the indirect and even long-term effects that are fundamental to generating a holistic view of an impact, are difficult to capture for a variety of reasons ranging from simply being unknown, to being abstract, qualitative and entirely theoretical. Furthermore, the SDGs are purposely designed with slight ambiguity, enabling each goal to be inclusive, interconnected, applicable to multiple stakeholders, and dynamic in order to absorb drastic global changes that may occur over the 15 years between their introduction in 2015, to their end goal in 2030 .

As a result, there is simply no standardised, one-size fits all method of measuring impact. Instead, there is a pluralism of impact measurement models proposed and used by different organisations. Having many different measurement methods then poses challenges to making comparisons within wide scope evaluations of multiple ideas, projects or policies, for example. For smaller-scale projects, impacts (positive or indirect) may be smaller and therefore easily ignored (for example, small scale economic impacts). Moreover, for these smallscale or start-up projects, there is often limited financial resource, along with many competing strains placed on the budget, therefore rendering impact measurement - which will require additional resource - a near impossible task. Finally, a fundamental difficulty in measuring impact is often that it is limited in and by design: it cannot easily be 'bolted on' as an afterthought. Concern for SDGs (and values) has to be an integral part of the design process, and this depends on design principles. 


\section{Design Perspectives}

In this section, the methodology of value-sensitive design is briefly reviewed, followed by our perspective(s) on the design of self-organising socio-technical systems with respect to a core set of critical human values: sustainability, socially-productive purposes, justice, legitimate governance, prosocial incentives, and personal identity. Based on this, we propose to enhance, or, complement the methodology of value-sensitive design (VSD) with a set of sets of inter-related design principles, which are applied depending on the perspective that is adopted.

\section{A. Value-Sensitive Design (VSD)}

In [1], it is suggested that VSD brings forward a "unique constellation of eight features", which includes: proactive influence of qualitative values on technological design from an early stage in the process; documenting values as "supra-functional" requirements with tests for compliance, system readiness and quality assurance; the iteration over and integration of conceptual, empirical and technical analysis and development; enlarging the scope of values beyond co-operation and participation (e.g. to self-actualisation and empowerment); distinguishing between usability and values with ethical significance; consideration of different classes of stakeholder, often observed in socio-technical systems; and building from the psychological proposition that values are universal (if possibly culturally relative).

However, we argue that socio-technical systems for solving wicked problems manifest many different values, and depending on the perspective one takes on the wicked problem that is being addressed, a different design approach is required.

\section{B. The Socio-Technical Systems "Necker Cube"}

We liken the problem to resolving a six-way Necker cube, because what is paramount depends, to a significant extent, on the perspective taken, which determines which face of the cube is 'on top', as illustrated in Fig. 1.
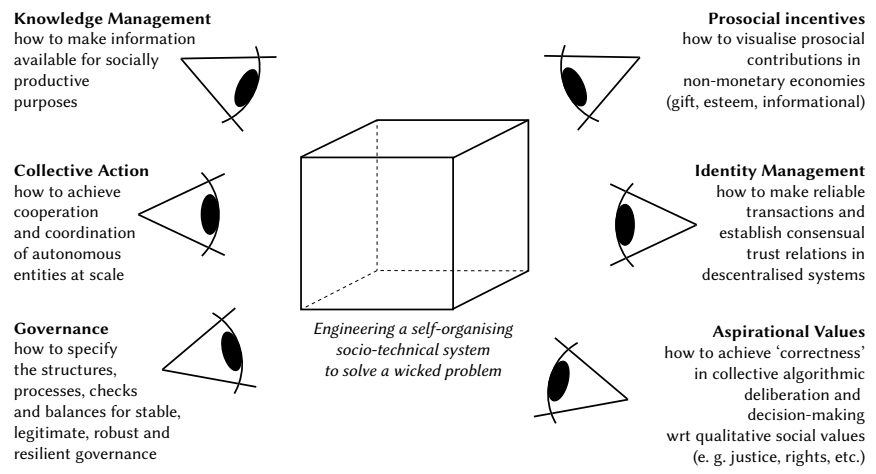

Fig. 1. The 6-Way "Necker Cube" for Value-Sensitive Design of Socio-Technical Systems.

Therefore, the design of a self-organising socio-technical system to address a wicked societal problem can be viewed as:

- a collective action problem: how provide the conditions for the evolution or emergence of cooperation and coordination of autonomous entities at scale;

- a knowledge management problem, how to make information available for socially productive purposes, from a diverse set of sources and expertise;

- a legitimate governance problem: how to determine the structures and processes for constitutional, collective and operational choice which avoid tyranny (as oligarchy, autocracy or majoritarian tyranny);
- a prosocial incentive problem, i.e. how to incentivise and visualise transactions in different types of non-monetary value-creation and exchange systems (e.g. esteem, gift, informational), to increase the social benefits of cooperation;

- an aspirational values problem, the ambition to achieve 'correctness' in collective algorithmic decision-making with respect to some set of shared and congruent values (aspirations); and

- an identity management problem, how to establish compartmentalised and consensual trust relations in decentralised systems, without compromising privacy.

\section{Design Principles}

In her pioneering work on self-governing institutions for sustainable common-pool resource (CPR) management [8], Ostrom observed that there were eight common features of the institution that determined whether or not the resource was sustained. She then turned to the issue of supply, and argued that if faced with a CPR collective action problem, instead of 'evolving' an institution with the necessary features, design one instead. The eight common features were then transformed into design principles. Effectively, these design principle represented deep social knowledge about the nature of self-governing institutions for sustain-able common-pool resource management.

In addition, in various works, we have attempted to identify similar findings from economic, political and social science on how the other problems (identified in the previous section) have been addressed in social systems, and we have tried similarly to distil this deep social knowledge into corresponding design principles. This has included:

- knowledge management principles derived in classical Athenian democracy [9], some of which are formalised in [10];

- principles of legitimate governance derived from a theory of basic democracy [11], and formalised as democracy by design [12];

- principles of prosocial incentives and social capital partially derived from anthropological studies of gift economies [13] and discussed as principles of axial (crytpo-)currency design [14]; and

- principles for aspirational values, in particular various different aspect of justice (e.g. distributive [15], retributive, procedural, and interactional).

The full set of sets of design principles is summarised in Table I. Note that the issue of identity management remains an open question, but design principles are being formulated, see e.g. [16].

However, even after applying all these principles in design, in practice there is also an inertial problem, caused by network growth as result of preferential attachment, the network effect (value increases non-linearly with scale), and the centralising tendency of the Internet at the application layer. This has inexorably led to the private ownership of the means of social coordination and information dissemination existing on an essentially publicly-built infrastructure. So there is a question of how to provide a viable alternative platform to the monopolist gatekeepers that have emerged as a result of the network effect at the application layer of the Internet.

We therefore need a platform which overcomes this inertial problem, supports multi-perspective VSD by encoding deep social knowledge (as captured by the design principles), and helps with impact measurement with respect to the SDGs. In fact, we need a meta-platform, a platform for generating platforms. A prototype of such a meta-platform is presented in the next section.

\section{PlatformOcean Meta-Platform}

Experience with digital platforms for eLearning, eHealth, etc., and for other 'as-a-service' operational models, would suggest that there is 
TABLE I. Design Principles for Socio-Technical Systems Collective Action

\begin{tabular}{c}
\hline Collective Action \\
Clearly defined boundaries \\
Congruence between rules and environment \\
Collective-choice arrangements respect self-determination Monitoring, by \\
self or appointed agencies \\
Graduated sanctions \\
Conflict resolution \\
Minimal recognition of right to self-organise \\
System of systems \\
Knowledge Management \\
Clearly defined boundaries \\
Lower transaction costs of knowledge exchange Agreement on common \\
interest questions \\
Distinction between common interest and partial goods Common \\
knowledge of procedural rules \\
Epistemic diversity \\
Recognition of expertise \\
Focal points for collective action
\end{tabular}

Legitimate Governance

Prevention rather than re-invention

Democracy is not an end-state, nor default Seamless transfer of power

No compromise on democratic processes Visibility, inclusivity, transparency, accountability Inter-dependence of diversity Education in pro-social benefits

Procedural evaluation and reflexivity

\section{Aspirational Values}

Clearly defined roles and powers

Educate - populate - majoritate

Create conditions for cooperation

Evaluate costs of enactment vs. non-enactment Publicity implies parity

Popularity does not imply impartiality Diversity of sources and forces Reflexivity

\begin{tabular}{c}
\hline Transactional Values \\
\hline Delimit purchasable goods \\
Identify purpose of currency \\
Closed loop (not convertible into fiat currency) Gratitude gift currency (not \\
judgement currency) Issuance (initialisation of currency) \\
Right to mint \\
Determination of allocation decided by guild No debt, no credit, no interest
\end{tabular}

so single universal technological solution suitable for all sustainabledevelopment collective-action problems. However, developing bespoke, and non-inter-operable platforms, leads to fragmentation, lack of re-use, loss of experience and ex-pertise, and so on.

Therefore, we are developing a meta-platform, which is being called PlatformOcean, which allows users to download an open source platform with a range of hosting options, instantiate that platform for particular requirements through mods and plug-ins, and make it accessible through a generic client and standard protocols.

The key design features of the PlatformOcean meta-platform, as illustrated in Fig. 2, are:

- deep social knowledge: the plug-in architecture also supports codification of the deep social knowledge captured by each of the five sets of design principles;

- generativity [17]: a tool to support the creation of new tools that were not envisaged by the designer of the original tool;

- common-pool development: the creation of plug-ins is a reflective process, as communities of developers use an instance of the platform to develop plug-ins for other instances;

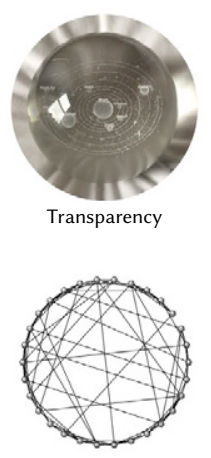

Decentralisation (many server options)

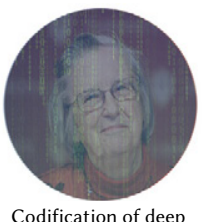
social knowledge
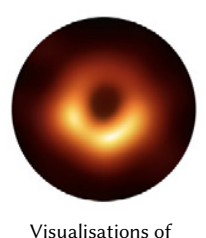
collective action
Codification of deep

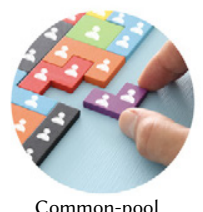

mon-poo development

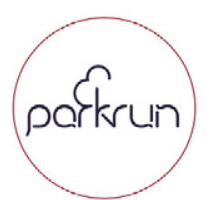

"Parkrunification" (see one; start one)

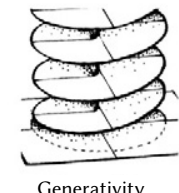

unexpected new tools)

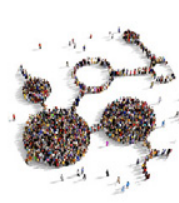

Ecosystem (PlatformOcean)
Fig. 2. PlatformOcean: Design Features.

- visualisation: for collective action, feedback to individuals and communities and how their small actions $X$ contributed to a greater action $\mathrm{Y}$ which had significant impact $\mathrm{Z}$;

- parkrunification: the generic platform can be used to create new downloadable instances of the platform, which others can use for faster customisation for related applications (we refer to this process as 'parkrunification' after the explosion of popularity of the parkrun phenomenon following the same process of observation and imitation);

- server-side transparency: the system architecture allows a range of options for self-hosting, with multi-purpose multi-function self-configuration implemented through plug-ins, supporting decentralisation;

- client-side transparency: each group or conversation in the client is with a different server, but open standard transfer protocols and programming interfaces provide seamless client-side integration, protect data, preserves privacy and prevent data leakage;

- ecosystem: the creation of a platform ecosystem supports sustainability through diversity and inhibits monopoly.

The PlatformOcean meta-platform provides a foundation for the codification and implementation of the design principles discussed in the previous section. The core idea is to provide communities with a fully customisable and self-hosted solution that best facilitates communication, whilst at the meta-level providing a toolset for collaboration in developing sustainable social media ecosystems. To achieve this aim, the meta-platform has been designed with a flexible plug-in architecture, inspired in part by other projects, for example: the Eclipse IDE, the computer game Minecraft, and Open Mustard Seed [18]. The overall architecture of PlatformOcean is illustrated in Fig. 3.

The platform is also designed to support client-side transparency. As Fig. 3 shows, each client conversation in a client-side app is interacting with one of the $\mathrm{n}$ platform instances, each of which can be distributed on a different platform with a different hosting option. Consequently, the server distribution appears seamless to the user.

The PlatformOcean meta-platform supports three additional features designed to overcome the inertial problem in the development and take-up of social platforms: self-customisation, generativity and re-use. The first of these key features is the flexible self-customisation and self-extension of individual PlatformOcean platform instances (see Fig. 3: UrbanRefill and UrbanForage platform instance are presented Section V and Section VI). Additionally, the open-standard nature of the communication protocol facilitates the development of custom clients, and increases interoperability and platform mobility. In this manner each platform instance, while derived from the same set of resources, acts in a way that is specialised to its user-base, and customised by its user-base. 


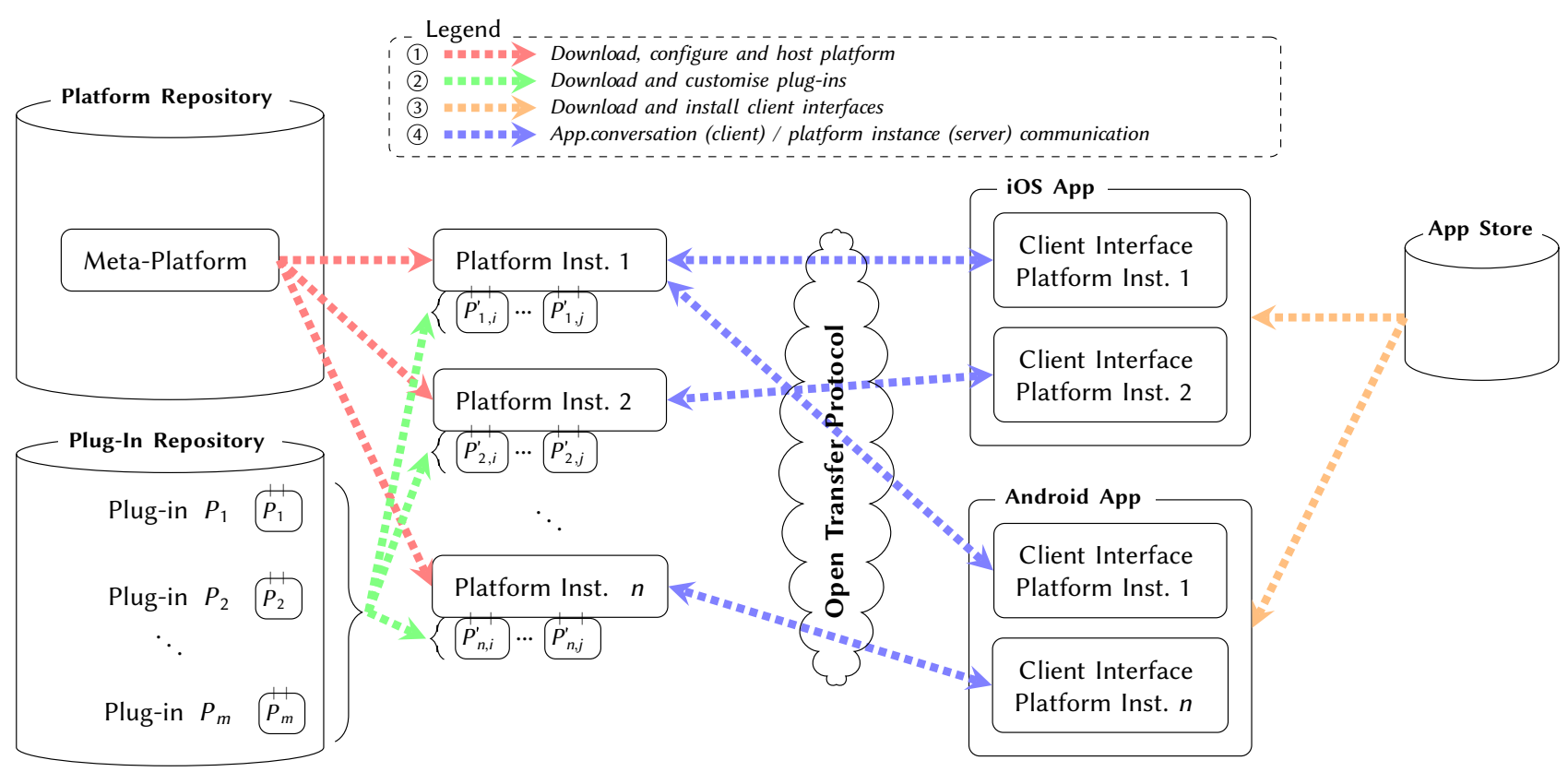

Fig. 3. PlatformOcean: Overall Architecture.

The plug-ins provide both additional functionality and the codification of deep social knowledge encapsulated by the design principles. Aspects of well-functioning governance systems - among others, the ability to implement deliberative assemblies based on rules of order [19], or allocate responsibilities fairly and define boundaries on member behaviour - can be replicated in server functionality using plug-ins. In the same way, plug-ins can also be designed to support the development of pro-social incentives, for example in the tracking and visualisation of progress towards goals over time or of individuals collaborating in a community to pool their achievements (as illustrated in the exemplars of the next two sections). Critically, we believe that re-usable plug-ins can also be developed for linking collective achievements to SDGs, and for linking platforms for different application which actually contribute towards SDGs in common. It is here that we envisage a particular role for AI components in a sociotechnical system, for example each plug-in could be a communicating agent in a multi-agent system, or could provide intelligent decision support for deliberative assemblies, or as a real driver for equity [20].

The second feature ties strongly with the 'meta' component of the meta-platform. PlatformOcean is instilled with a notion of generativity. By sharing plug-ins and client designs, PlatformOcean aims to facilitate an over-arching social media eco-system, wherein disparate groups can support each other through the de-velopment of 'reusable parts'. A central plug-in and client repository (as illustrated in Fig. 3) will ideally provide a hub for shared development. This calls back to the concept of social capital: this central hub may have the added benefit of providing opportunities for previously unconnected groups to meet, 'build bridges' and collaborate on projects outside of the PlatformOcean context.

The final feature refers to the accessibility of the platform. The ease of setting up and self-hosting an instance of PlatformOcean is a key consideration. The server software can be installed, customised and run on hardware ranging from raspberry pi to personal computers with fairly minimal technical knowledge required. As technical solutions increase in complexity, the skills often required to set up and maintain them can often increase. Keeping the platform accessible to those with little technical background is imperative to its function as a sustainable solution. Moreover, the aim is to create open source archives and encourage collective development of plug-ins, i.e. this is a collective action problem in itself, and could be addressed by an instance of PlatformOcean itself (see Fig. 4).

The next two sections present the design of two such platform instances to address an environmental and a societal problems, respectively excess plastic waste (Section V) and unequal food distribution (Section VI).

\section{Exemplar 1 - Plastic Waste Reduction}

In this section, we describe the design of an app called UrbanRefill, aiming to reduce the use of plastic (and increase of plastic waste) by reusing liquid containers. The design of UrbanRefill applies the design principles of Section III.C with the target meta-platform of Section IV, addressing the following requirements:

- functional requirements: achieve the basic function of the application by reducing plastic consumption;

- value-sensitive requirements: enhancing sustainability, improving inter-connectivity and community capability for collective action;

- SDG-sensitive requirements: wider contribution to protecting the planet; preventing climate breakdown and; achieving the relevant SDGs.

We address each of these requirements in turn.

\section{A. UrbanRefill: Application Design}

The universal abundance of plastic has caused significant and wide scope damage to the planet, involving issues such as microplastic pollution and increased waste, leading to (sometimes irreversible) damage to numerous ecosystems. Additionally, our current plastic use fuels an unsustainable throwaway culture, in which the majority of products are deemed dispensable.

PlatformOcean's UrbanRefill application focuses on reducing single use plastics, by enabling the refilling of common household products, such as washing up liquid, hand soap and shampoo, as opposed to the purchase of new ones. In the UK at present, there are several types of refill scheme; however these have some limitations, ranging from failures to design practicality into the scheme, whilst also resulting in higher transport-related emissions (e.g. by using heavier materials such as glass), to an over-reliance on people's organisational skills. 


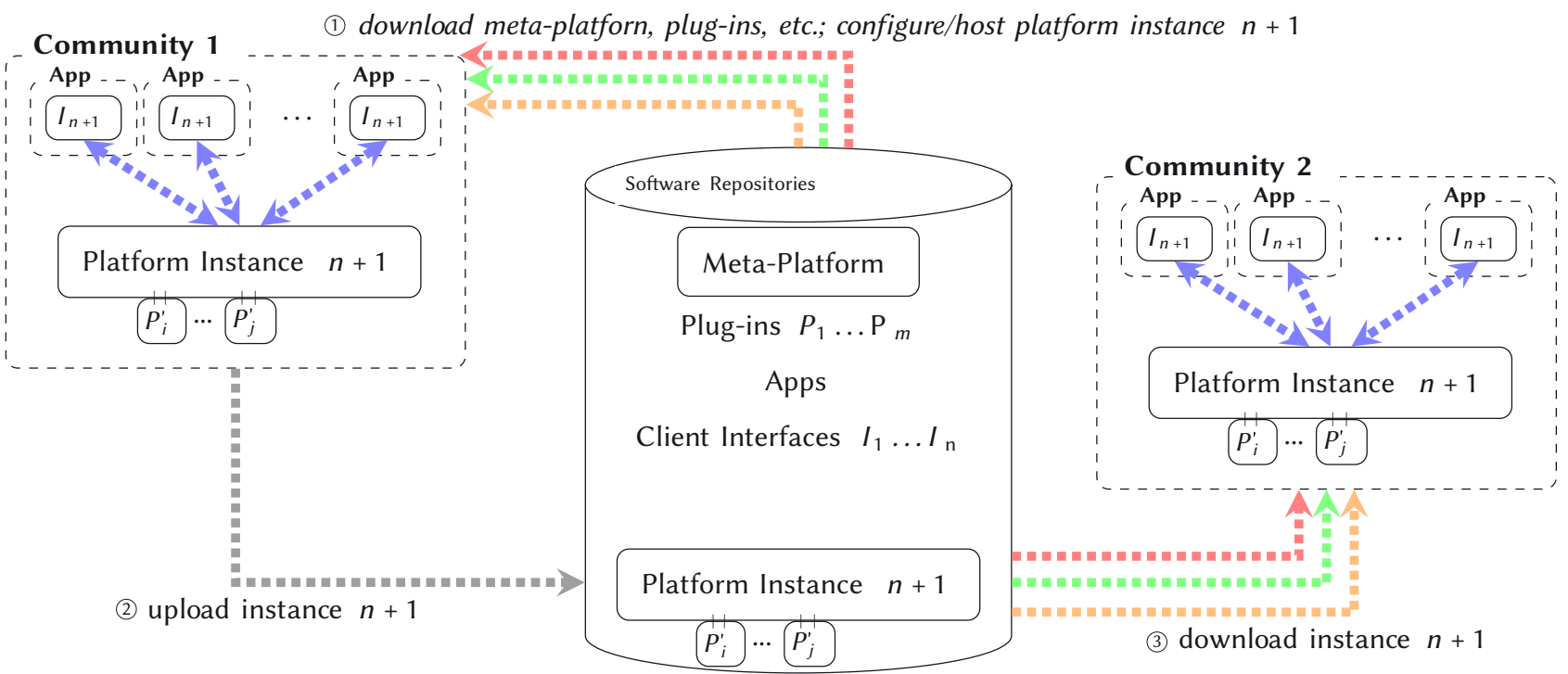

Fig. 4. PlatformOcean: Timeline of Re-use.
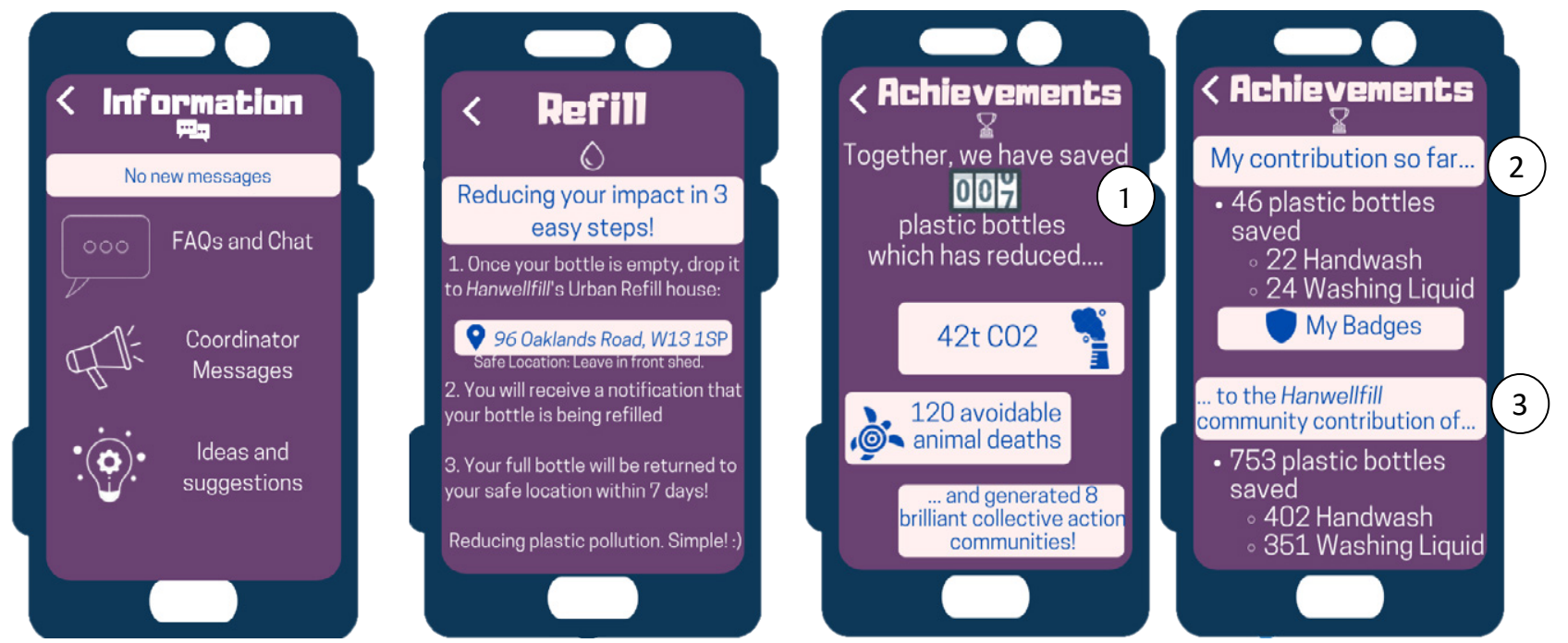

Fig. 5. UrbanRefill Design Template. Showing four screens (from left to right): Information which encourages community-wide active engagement, thus satisfying collective-choice arrangement principles, as well as visibility, inclusivity, transparency and accountability principles; Refill which provides Members with common knowledge of the rules; and Achievements, visualising community contribution to promoting values (e.g. sustainability) and to achieving SDGs.

UrbanRefill is distinct from other refill schemes in that it generates a community-led initiative to refill ${ }^{1}$. The premise is straightforward: within a given community setting, one Coordinator holds products in bulk, with Members dropping empty bottles off for the Coordinator to then refill and return within seven days. Importantly, for each product, there is a generic type of bottle which has a unique barcode and is associated to a particular Member. The app supports the management, coordination and logistical elements of the refill process in several ways:

- A Member's Activity Status provides information on the receipt of an empty bottle, as well as its return. Additionally, Members can choose to opt-in to push notifications for this information.

- The Coordinator has access to a bottle barcode scanning feature, which is used to scan empty bottles in, thereby marking them as refill in progress. The associated Member's bottle Activity Status is updated, and a Coordinator Workflow is generated, enabling the Coordinator to keep a track of the seven-day turnaround.

${ }^{1}$ Currently the focus is on the aforementioned household goods, but variations of the application could enable expansion into many more products, as well as different ways of organising individuals within the community.
- In addition, the Coordinator has access to a map which reflects the location of Members and their bottle activity status, thus aiding the management and logistics of returns.

- Due to the genericity of the bottles for each product, prices and payments are pre-approved by Members and automatically taken upon the Coordinator marking a bottle as returned.

\section{B. Applying the Design Principles}

A quartet of screenshots of UrbanRefill are shown in Fig. 5, illustrating aspects of functional, value-sensitive and SDG-sensitive design. This section discusses each of these in turn.

\section{Functional Design}

With respect to the functioning of UrbanRefill (i.e. achieving the basic function of the app), there is the capability to ensure congruence between the rules and the environment, as the bottle drop off activities of both the Members and Coordinator can be individually configured according to the abilities of the people involved in the task. In addition, as the system is designed to take place within a local community setting, the transaction costs are lowered as these 
drop-off activities can be incorporated into tasks that were already occurring. Finally, with respect to the design principle of publicity implies parity, both Members and Coordinators have access to essential knowledge within the app, with the opportunity to gather additional information through the use of the FAQ or Chat systems.

\section{Value-Sensitive Design}

There are a number of design principles that have been utilised in the designing of UrbanRefill to address the value-sensitive requirements of the app. Firstly, there are clearly defined boundaries within the community - a participant is either a Member or a Coordinator and the rules for those who have the right to appropriate from the common pool resource are correspondingly clearly defined, according to the design principle of common knowledge of rules and their generation.

Secondly, through the use of the Information page, collectivechoice arrangements are encouraged, as members are able to provide feedback on the functioning of the system, whilst also contributing to the expansion and growth of the products that are available within their community.

Thirdly, reflexivity within the community is designed with a Feelings response that can be completed by both Members and Coordinators after each transaction. Aggregating this data within a community provides a visualisation of overall satisfaction, enabling introspection on the collective endeavour. This information could also prove useful in future variations with respect to dispute resolution (see below).

Finally, visibility, inclusivity, transparency and accountability are well-woven into the design of UrbanRefill, particularly within the Information page which aids transparency, inclusivity and active engagement throughout the community on past and current decisions. Transparency and visibility are also achieved as all Members are aware of the Coordinator being the primary decision-maker, with inclusivity also encouraged within this process. Additionally, designed into the basic premise of the app are well-established rules for the group, for example, regarding the mechanisms and frequency of refilling. The design of group achievements within the Achievements page will also aid in encouraging each community to work collectively.

\section{SDG-Sensitive Design}

By designing in a clearly established common interest question - achieving the goal of reducing plastic waste - UrbanRefill's Achievements page enables the aims of the ecosystem to be achieved. In doing so, positive contributions made towards SDGs are visible for all Members and Coordinators, which not only encourages use of the app but, crucially, allows for the im-pact of these positive contributions to be measured. By including the Achievements page within the design of UrbanRefill, impact measurement can occur. The contribution that UrbanRefill makes towards achieving the SDGs, and how this impact could be measured, is discussed in Section V.C.

\section{Limitations}

However, some of the identified principles are missing, either because they are not applicable, or because the user-centred design process could not anticipate the problems that could occur. For example, design principles relating to graduated sanctions and conflict resolution have not (yet) been included and are currently not applicable, as these 'errors' or misuses of the app are learned from trial and real-world implementation. In addition, the design of axial-crypto currencies (prosocial incentives) are not applicable, and design has been omitted, due to the fact that the current version of UrbanRefill uses only real-world financial transactions for real-world products.
It is, however, very possible that in other variations of the app such principles will need to be considered - for example in a case in which each Member harbours one of the products, with exchange done between Members on a product-equivalence basis rather than a financial exchange. Finally, principles of democracy by design (legitimate governance) are mostly absent from the current version of the app as it does not wholly function as a rules-based system, particularly with respect to the power balance between Members and Coordinators. Despite designing in both structures for organising the scheme and encouragement to produce an equal and fair system with less centralised authority (e.g. encouraging engagement of Members on decision making through the Coordinator Messages), the Coordinator does appear to control the majority of decisions. Therefore, as the system would scale, it might be necessary to introduce an 'Ombudsperson' role who would intervene in dispute resolution.

\section{Impact on SDGs}

Whilst, at present, there is not a focussed SDG designed to target the issues surrounding anthropogenic plastic generation and consumption specifically, given our ubiquitous and varied use of this material, the potential threats and present damage it causes, and its near "indestructible" nature (microplastics takes hundreds of years to degrade), the issue of plastic pollution spans several of the 17 SDGs. The predominantly relevant SDGs identified to plastic use are highlighted below, alongside a brief explanation as to how the use of UrbanRefill will contribute to the achievement of the specified goal, as well as, crucially, how this impact can be measured and reflected within the Achievements section of the app.

\section{SDG 11: Sustainable Cities and Communities}

Impact: building community networks that both allow for and promote sustainability, whilst also reducing plastic waste, which is often shipped from developed to developing countries;

Measurement: monitoring the number of Refill communities built on the platform can contribute to better understand the number of ongoing community-lead collective action initiatives.

\section{SDG 12: Responsible Consumption and Production}

Impact: generating a cultural and societal change to no longer accept the unsustainable norms of plastic use;

Measurement: tracking the number of plastic bottles 'saved' (i.e. not used because the bottle has been reused), and therefore the number of times individuals have chosen to reuse, rather than repurchase.

\section{SDG 13: Climate Action}

Impact: reducing plastic use lowers the emissions associated with plastic production processes, and with subsequent delivery of these goods to customers;

Measurement: estimating the total equivalent $\mathrm{CO} 2$ emission reduction as a result of the number of bottles 'saved'. This could also be disaggregated to display the information at individual, community and ecosystem levels.

\section{SDG 14: Life Below Water}

Impact: reducing plastic pollution in bodies of water, thereby lowering the consumption by fish, animals caught in waste materials, and microplastic chemical pollution;

Measurement: estimating the number of animal lives saved from death incurred due to plastic consumption or entanglement.

\section{SDG 15: Life on Land}

Impact: reducing plastic pollution on land and lowering the build-up of non-recyclable plastics, reducing its consumption by birds and other land animals;

Measurement: estimating the number of animal lives saved from death 
incurred due to plastic consumption, as well as the number of plastic bottles 'saved' and not contributing to waste build-up.

With regards to SDG 3 and 4, the use of UrbanRefill will undoubtedly contribute positively to these SDGs, however, no metric has yet been identified to accurately reflect the impact that the use of UrbanRefill has made towards achieving the goal.

\section{SDG 3: Good Health and Wellbeing}

Impact: by preventing climate change, wellbeing is likely to improve, generating or improving a community network can encourage active transport and improve wellbeing.

\section{SDG 4: Clean Water and Sanitation}

Impact: reducing microplastic pollution which affects all waterways including freshwater bodies, and thereby preventing chemical decomposition of plastics within freshwater.

Finally, it is important to note that, as mentioned in Section II, one of the challenges of interconnectedness and impact measure-ment is being aware of, and measuring unintended impact. It is an 'unknown unknown' that would need to be anticipated in any evaluation of UrbanRefill and the focal point of any re-design.

\section{Exemplar 2 - Surplus Food Redistribution}

This section describe the design of a second app, called UrbanForage. The aim of UrbanForage is to decrease food waste through surplus food distribution, and again its design applies the principles of Section III. C and targets the meta-platform of Section IV, in particular with plug-ins delivering common functionality.

\section{A. UrbanForage: Application Design}

Food loss and waste has become one of the most pressing resourceuse challenges at community, national and international scales. Globally, a third of all food produced for human consumption is either lost or wasted [21], resulting in the additional loss of all embodied inputs such as water, energy, labour, land and capital. Food waste accounts for $8 \%$ of global anthropogenic greenhouse gas emissions and costs the global economy $\$ 940$ billion annually [22], [23]. Not only are the environmental and economic impacts significant, there are also huge ethical consequences, as 1 in 9 people remain undernourished in a world where excess food is generated on a daily basis [22]. The redistribution and reuse of surplus food is a means to reduce the impacts of food waste, which is arguably a product of the current unsustainable food supply chain. However, as well as seeking systemic change through political channels, we can also try to apply pressure through bottom-up behavioural change.

In the UK, surplus food redistribution and reuse charities are having a profound impact at both local and national levels. Collectively, these charities work across all sectors of the food system, in addition to multiple community level initiatives and digital applications. However, at least three limitations can be identified. Firstly, not all surplus food donations are either environmentally or economically viable for collection by surplus food charities. Secondly, user retention for relevant applications is not consistent, thus labour to do the necessary work is not always guaranteed. Finally, the independent and fragmented nature of current community level efforts reduces the true impact potential of collective community action [24].

The UrbanForage application brings together four potential stakeholders:

- Coordinator: a charity such as City Harvest ${ }^{2}$ or The Felix Project ${ }^{3}$ who acts a broker people the other three stakeholders;

\footnotetext{
2 www.cityharvest.org.uk/

3 thefelixproject.org
}

- Surplus food donors: organisations with excess food, e.g. supermarkets, restaurants, wholesalers, caterers, etc.;

- Beneficiaries: organisations providing nourishment to vulner-able people, such as homeless shelters, women's refuges, care centres, children's breakfast clubs, etc.;

- Volunteers: individuals providing pick-up and delivery.

In particular, UrbanForage addresses the first limitation, that some pick-ups and drop-offs are uneconomic or non-environmental to use a van, so we try to use volunteers who walk or cycle, and lower transaction costs by aligning the delivery route with a journey they would have taken anyway, for example as part of their commute. In this way UrbanFor-age enhances the capacity of redistribution charities through increasing stakeholder self-organisation.

\section{B. Applying the Design Principles}

Despite the apparent differences in functionality, many of the issues addressed by UrbanForage are the same as in UrbanRefill; therefore generic plug-ins can be used to provide this functionality: a notable example is the Badges and Impacts pages. Moreover, the client interface has much in common, so unsurprisingly then perhaps, the look-and-feel of both applications is similar, see Fig. 6 .

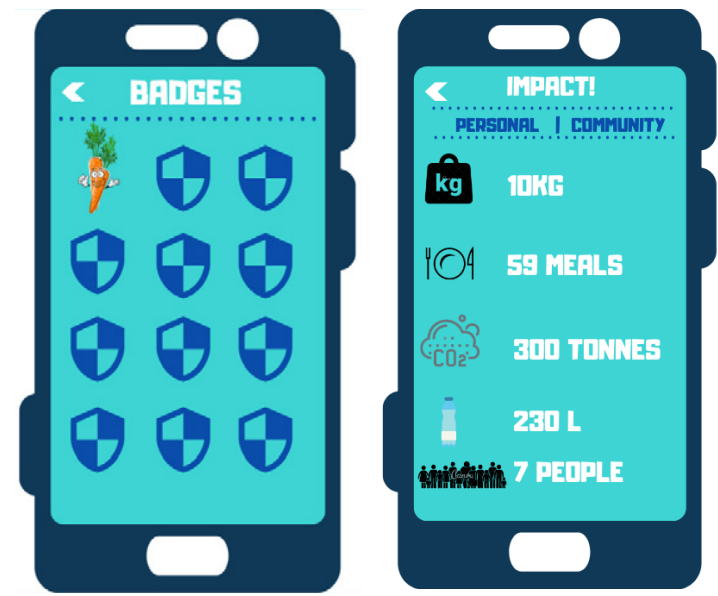

Fig. 6. UrbanForage: Badges and Impact Screens.

However, there are key divergences as well. One notable divergence is in the monitoring and sanctions, which as noted above, in UrbanRefill, was left undesigned, but in UrbanForage the volunteers need to be vetted by the coordinating charity (a legal responsibility) and moreover their performance of delivery tasks has to meet an approved standard (food delivered too late is still wasted; some food types deteriorate, and so on). Therefore, the system of monitoring and sanctions has to be very explicit, but for this purpose, a bespoke plugin can be designed and implemented, and integrated like any other plug-in.

\section{Impact on SDGs}

The current unsustainable and resource-intensive food system results in vast amounts of food loss and waste. Due to the interconnected nature of the food system, its impacts -environmental, economic, social and political - are complex, having both direct and indirect impacts on a number of SDGs. Several of these are in common with the UrbanRefill application, but the relevant SDGs identified to food loss and waste are highlighted below. We give a brief explanation as to how the use of UrbanForage will contribute to the achievement of the specified goal, as well as, crucially, how this is impact can be measured and reflected within the Achievements page of the app. 


\section{SDG 2: Zero Hunger}

Impact: the redistribution and reuse of surplus food facilitates meal generation for the most vulnerable, as well as adds to food bank resources, helping to mitigate against hunger and food insecurity;

Measurement: monitoring the number of meals provided can provide figures as to how many vulnerable people are receiving regular meals.

\section{SDG 12: Responsible Consumption and Production}

Impact: redistributing and reusing surplus food directly de-creases the quantity of food waste;

Measurement: monitoring the net amount of surplus food that is reused will provide data as to how much food waste is prevented.

\section{SDG 13: Climate Action}

Impact: a reduction in food waste directly decreases emissions associated with embodied input (e.g. land, water, energy), as well as emissions generated in landfill;

Measurement: estimating equivalent water, $\mathrm{CO} 2$ emission and net weight of food resource saved; and aggregating this data across multiple local charities and food distribution schemes into national statistics.

\section{SuMMARY AND CONCLUSIONS}

No man is an island, entire of itself

- John Donne, 1624

In summary, the basic problem addressed by this paper is engineering socio-technical systems to address wicked societal problems and contribute to meeting the UN Sustainable Development Goals. It presented six different perspective on the problem and, for five of them proposed a set of design principles. Based on the reconfigurable plugin architecture of the meta-platform (PlatformOcean), it was proposed to use AI in plug-ins, firstly to codify the deep social knowledge encapsulated by the principles to support organisational coherence with respect to the SDGs, and secondly to facilitate the collection of data for measuring and assess-ing impact on the SDGs.

In this sense, this work could be considered to be an instantiation of value-sensitive design called Sustainable Development Goal-Sensitive design. We have applied this design methodology applications for two pressing societal problems, excess plastic waste and unequal food distribution. In both cases, it could be seen how individual data and actions could be aggregated and processed by AI plug-ins in order to assist with self-organisation and sustainability but also to visualise individual contributions to the collective and make recommendations.

As we transition further towards the Digital Society and progress towards reaching the SDGs' 2030 target, there is a unique opportunity - and a profound responsibility - to consider what sort of digital future we want to shape.

In particular, we have argued that socio-technical platforms for social coordination are not standalone systems, and have to be designed and deployed with respect to each other as part of a "platform ecosystem", with respect to qualitative human values, and the impacts on the SDGs. The particular role of AI anticipated here would be variously in the coordination and sustainability of the ecosystem, in the codification of deep social knowledge that helps realise human values, and in the oblique measurement of the impact on SDGs (see, for example, [25]).

In measuring these impacts, we are able to account for the wider implications of our digital innovations, and by doing so, we are provided with immense prospects: mitigating anthropogenic climate change, reducing inequality and poverty, and promoting peace and justice. Through measuring the impact of our plans, projects and policies against the UN SDGs, we are offered the ability to assess our behaviour against the universally agreed goals that form the framework of our future.

PlatformOcean, and the two exemplars shown within this paper, have been designed not just with functionality in mind, but also considering both the intended positive and unintended impacts towards the SDGs. In particular, the design of plug-ins, and the use of computational intelligence (AI) within those plug-ins, could provide the basis for effective human-machine interaction and cooperation in socio-technical systems: on the one hand through the codification of deep social knowledge and self-organisation for sustainability, for example; and on the other hand measurement, impact assessment and feedback which contributes to the achievement of (or trade-off between) SDGs.

Future work must now strengthen the bridge the gap between digital innovation and achieving the SDGs, and using AI to measure impact is an important first step. Everyone and everything is interconnected: we can no longer hide from the wider impacts that our individual actions, or the algorithms that we develop, have on society, or the planet [26]. The Digital Society is everyone's future - but as everyone and everything is interconnected, that interconnection should be leveraged for the common interest of the collective, not for the personal profit of a privileged few.

\section{ACKNOWLEDGMENT}

We are grateful to the Association for Information Technology Trust (AITT) for support for project PlatformOcean. We also thank the organisers of the ECAI2020 AI4Eq Workshop, and the anonymous reviewers of various submissions for their many encouraging comments and helpful recommendations.

\section{REFERENCES}

[1] B. Friedman, P. Kahn, A. Borning, "Value sensitive design and information systems," in The Handbook of Information and Computer Ethics, K. Himma, H. Tavani Eds., Wiley, 2008, pp. 69-101.

[2] K. Bellman, J. Botev, H. Hildmann, P. Lewis, S. Marsh, J. Pitt, I. Scholtes, S. Tomforde, "Socially-sensitive systems design: Exploring social potential," IEEE Tech. Soc. Mag., vol. 36, no. 3, pp. 72-80, 2017.

[3] H. Rittel, M. Webber, "Dilemmas in a general theory of planning," Policy Sciences, vol. 4, no. 2, pp. 155-169, 1973.

[4] S. Zuboff, "Big other: surveillance capitalism and the prospects of an information civilization," Journal of Information Technology, vol. 30, pp. 75-89, 2015.

[5] H. Simon, "Rational choice and the structure of the environment," Psychological Review, vol. 63, no. 2, pp. 129-138, 1956.

[6] T. Hák, S. Janoušková, B. Moldan, "Sustainable development goals: A need for relevant indicators," Ecological Indicators, vol. 60, pp. 565 - 573, 2016.

[7] Common Fund for Commodities, "The sustainable develop-ment goals and impact measurement - a CFC journey." Annual Report, 2017.

[8] E. Ostrom, Governing the Commons. Cambridge Univ. Press, 1990.

[9] J. Ober, Democracy and Knowledge. Princeton Univ. Press, 2008.

[10] D. B. Kurka, J. Pitt, J. Ober, "Knowledge management for self-organised resource allocation," TAAS, vol. 14, no. 1, pp. 1:1-1:41, 2019.

[11] J. Ober, Demopolis: Democracy before liberalism in theory and practice. Cambridge Univ. Press, 2017.

[12] J. Pitt, J. Ober, "Democracy by design: Basic democracy and the selforganisation of collective governance," in IEEE SASO, 2018, pp. 20-29.

[13] B. Malinowski, Argonauts of the Western Pacific: An account of native enterprise and adventure in the Archipelagoes of Melanesian New Guinea. Routledge and Kegan Paul, 1922.

[14] J. Pitt, J. H. Clippinger, C. Sørensen, "Values, axial curren-cies, and computational axiology: Digital currencies can do more than buy stuff," IEEE Technol. Soc. Mag., vol. 37, no. 3, pp. 56-63, 2018. 
[15] N. Rescher, Distributive fustice. Bobbs-Merrill, 1966.

[16] A. Rychwalska, G. Goodell, M. Roszczynska-Kurasinska, "Data management for platform-mediated public services: Challenges and best practices," CoRR, vol. abs/1909.07143, 2019.

[17] J. Zittrain, The Future of the Internet - And How to Stop It. New Haven, CT: Yale University Press, 2008.

[18] T. Hardjono, P. Deegan, J. Clippinger, "Social use cases for the ID3 open mustard seed platform," Technology \& Society Magazine, vol. 33, no. 3, pp. 48-54, 2014.

[19] S. Robert, H. Robert, W. Evans, D. Honemann, T. Balch, Robert's Rules of Order, Newly Revised, 10th ed. Perseus Publishing, 2000.

[20] A. Manjarrés, A. Pickin, M. A. Artaso, E. Gibbons, "Ai4eq: for a true global village not for global pillage," Technology \& Society Magazine, vol. 40, no. 1, 2021

[21] Food and Agriculture Organisation of the UN, "Global food losses and food waste - extent, causes and prevention." http://www.fao.org/3/ mb060e/mb060e00.pdf.

[22] Food and Agriculture Organisation of the UN, "Food wastage footprint and climate change." http://www.fao.org/3/a-bb144e.pdf.

[23] C. Hanson, P. Mitchell, The Business Case for Reducing Food Loss and Waste. Washington, DC: Champions 12.3, 2017.

[24] E. Facchini, E. Lacovidou, J. Gronow, N. Voulvoulis, "Food flows in the united kingdom: The potential of surplus food redistribution to reduce waste," Journal of the Air \& Waste Management Association, vol. 68, no. 9, pp. 887-899, 2018.

[25] D. Schiff, B. Rakova, A. Ayesh, A. Fanti, M. Lennon, "Explaining the principles to practices gap in AI," Technology \& Society Magazine, vol. 40, no. 1, 2021

[26] A. Luccioni, Y. Bengio, "On the morality of artificial intelligence," IEEE Tech. Soc. Mag., vol. 39, no. 1, pp. 16-25, 2020.

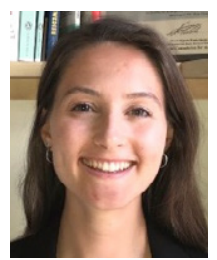

Steph Pitt

After graduating with a BSc in Natural Science from the University of Bath (UK) in 2019, Steph worked on air quality research in North East Asia as an Environmental Affairs Intern at the United Nations ESCAP office in South Korea. Subsequently, she worked on improving social coordination within local plastic reduction and air quality movements as part of the PlatformOcean project at Imperial College London. Currently, Steph is studying for a Master's in Public Health Science at Karolinska Insitututet in Sweden, with a focus particular interest on the intersection between public health interventions and environmental sustainability.

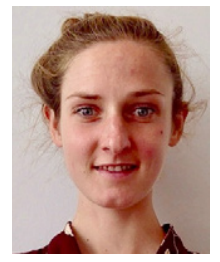

Marlína van Meelis Lacey

Marlína van Meelis Lacey graduated with a BSc in Environmental Geography and International Development from The University of East Anglia (UK). She has worked in Cambodia for two NGOs: The Human Resource and Rural Economic Development Organisation (HURREDO) and Youth Coalition for Unity and Development (YCUD), and for the Raleigh Interna-tional Water, Sanitation and Hygiene (WASH) programme based in India. She has also worked as a drone pilot for an AI start-up and on the PlatformOcean project at Imperial College London.

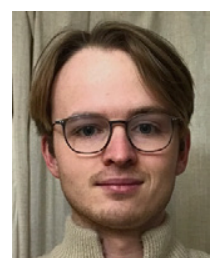

\section{Ed Scaife}

Ed Scaife graduated with an MEng ACGI in Electronic and Information Engineering from Imperial College London (UK) in 2020. He was the principal developer of the prototype for the sustainable social media meta-platform PlatformOcean. Outside of this, his main academic interests are Computer Vision, Artificial Intelligence and HumanCentred Robotics. He currently works as a software and systems engineer in the automotive industry.

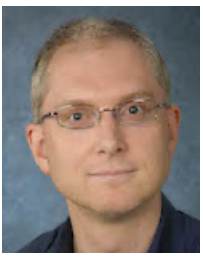

Jeremy Pitt

Jeremy Pitt is Professor of Intelligent and Self-Organising Systems in the Depart-ment of Electrical and Electronic Engi-neering at Imperial College London (UK). His research interests focus on developing formal models of social processes using computational logic, and their application in self-organising multi-agent systems for engineering cyber-physical and socio-technical systems. He has been an investigator on more than 30 national and European research projects and has published more than 150 articles in journals and conferences. He is a Fellow of the BCS and a Fellow of the IET, a member of the IEEE, and since 2018 has been Editor-in-Chief of IEEE Technology and Society Magazine. 